\title{
CARACTERÍSTICAS SOCIODEMOGRÁFICAS DE ADOLESCENTES QUE VIVEM COM familiares alcoolistas no município de João Pessoa, Paraíba
}

\author{
Vagna Cristina Leite da Silva Pereira ${ }^{1}$ \\ Lorena de Farias Pimentel Costa ${ }^{2}$ \\ Estela Rodrigues Paiva Alves ${ }^{3}$ \\ Camila Abrantes Cordeiro ${ }^{4}$ \\ Luanna Silva Braga ${ }^{5}$ \\ Maria de Oliveira Ferreira Filha ${ }^{6}$
}

\begin{abstract}
Tem-se como objetivo estimar o quantitativo de adolescentes que habitam lares com familiares alcoolistas em uma amostra representativa de adolescentes na cidade de João Pessoa, Paraíba, e caracterizar os perfis sociodemográficos e familiares dos adolescentes que convivem com familiares alcoolistas. Estudo do tipo transversal de base populacional, realizado com 715 adolescentes entre 14 e 19 anos. Foram utilizados questionário sociodemográfico e o CAGE-familiar. Para análise dos resultados, foi utilizada estatística descritiva. De acordo com resultados, a maioria dos adolescentes eram meninas, com idade de 17 anos; entre os familiares alcoolistas, o pai foi mais referenciado. Torna-se necessário implementar ações de prevenção para a saúde dos adolescentes com familiares alcoolistas, levando em consideração o risco para o desenvolvimento de problemas biopsicossociais.
\end{abstract}

Descritores: Alcoolismo; Relações Familiares; Adolescente.

\footnotetext{
${ }^{1}$ Doutoranda, Universidade Federal da Paraíba, João Pessoa, PB, Brasil. Professor, Faculdade de Enfermagem Nova Esperança, João Pessoa, PB, Brasil.

2 MSc, Professor Auxiliar, Universidade Federal de Campina Grande, Campina Grande, PB, Brasil.

${ }^{3}$ Doutoranda, Universidade Federal da Paraíba, João Pessoa, PB, Brasil. Pesquisador, Universidade Federal da Paraíba, João Pessoa, PB, Brasil. Pesquisador, Universidade de Pernambuco, Recife, PE, Brasil.

${ }^{4}$ MSc.

${ }^{5}$ MSc, Professor Substituto, Universidade Estadual da Paraíba, João Pessoa, PB, Brasil.

${ }^{6}$ PhD, Professor, Universidade Federal da Paraíba, João Pessoa, PB, Brasil.
} 


\section{SOCIODEMOGRAPHIC CHARACTERISTICS OF ADOLESCENTS LIVING WITH alcoholic families in the city of João Pessoa, Paraíba}

The aim of this study is to estimate the amount of teens that inhabit homes with alcoholic families in a representative sample of adolescents in the city of João Pessoa, Paraíba; then characterize the demographic profiles of these adolescents and their alcoholic families. This is a cross-sectional population-based study, conducted with 715 teenagers between the ages of 14 to 19 years old. A sociodemographic questionnaire and CAGE-family instrument were used. For data analysis, descriptive statistics were used. According to the results, most of the teenagers were girls, age 17 years old, and within the alcoholic family, the father was most referenced. It is necessary to implement preventive measures for the health of teens with alcoholic family members, taking into account the risk for the development of biopsychosocial problems.

Descriptors: Alcoholism; Family Relations; Adolescent.

\section{Características SOCIOdemográficas de ADOLESCENTES QUE VIVEN CON familiares alcohólicos en el municipio de João Pessoa, Paraíba} Se tiene como objetivo estimar cuantitativamente los adolescentes que habitan en hogares con familiares alcohólicos, en una muestra representativa de adolecentes en la ciudad de João Pessoa, Paraíba, y caracterizar los perfiles sociodemográficos y familiares de los adolescentes que conviven con familiares alcohólicos, es un estudio transversal de base poblacional realizado con 715 adolescentes entre los 14 y los 19 años. Fueron utilizados cuestionario sociodemográfico y el CAGE-familiar. Para el análisis de resultados se utilizó la estadística descriptiva. De acuerdo con los resultados, la mayoría de los adolecentes eran chicas de 17 años, ente los familiares alcohólicos el padre fue el mas referenciado. Es necesario implementar acciones de prevención para la salud de los adolescentes con familiares alcohólicos, teniendo en cuenta el riesgo para desarrollar problemas biopsicosociales.

Descriptores: Alcoholismo; Relaciones Familiares; Adolescente.

\section{Introdução}

Durante a evolução histórica da sociedade, a ingestão de substâncias psicoativas manteve-se regulada dentro de contextos socioculturais específicos que condicionavam o consumo a partir de padrões e normas próprias de cada cultu$\mathrm{ra}^{(1)}$. Porém, a sociedade atual se defronta com o aumento no consumo de substâncias entorpecentes lícitas ou ilícitas que se torna, a cada dia, um problema de saúde pública mundial, levando em consideração que, a cada ano, cerca de 40\% da população, o que corresponde a, aproximadamente, 2 bilhões de pessoas, fazem uso abusivo de bebidas alcoólicas ${ }^{(2)}$.

O Ministério da Saúde também faz afirmações de alerta quando expõe que os transtornos mentais em decorrência do abuso de álcool e de outras drogas exigem atendimento regular para cerca de $6 \%$ a $8 \%$ da população brasileira, 
embora ainda existam estimativas mais elevadas ${ }^{(3)}$. Nesse caso, a dependência química é vista como um problema social que causa deterioração da vida mental, orgânica e social do indivíduo, além de prejudicar, claramente, suas relações interpessoais e desestruturar a convivência familiar ${ }^{(4)}$.

Para tanto, a Organização Mundial de Saúde afirma que a dependência química deve ser tratada como doença médica e problema social, à medida que se caracteriza como um estado mental e físico simultaneamente. Isso acontece devido à interação do indivíduo com a substância, gerando compulsão pelo consumo, relacionada aos efeitos psíquicos e desconforto provocado pela ausência da mesma. Nesse caso, é preciso, além de identificar e tratar os sintomas, conhecer as causas e consequências da dependência, com o intuito de promover novas possibilidades para mudança de comportamento ${ }^{(5)}$.

A dependência química também é problemática em relação à motivação para o início do tratamento, principalmente, no que se refere ao alcoolismo, levando em consideração o desenvolvimento lento para a instalação da dependência associada à forte aceitação social que a bebida alcoólica tem. Esses fatores dificultam a identificação do momento em que o sujeito deixa de ser um "bebedor social" e torna-se um dependente $^{(6)}$.

$\mathrm{Na}$ atualidade, a problemática do álcool vem tomando grandes proporções sociais, levando em consideração o elevado grau de prejuízos que o alcoolismo oferece, principalmente, quando se refere ao alcoolista e à sua relação familiar, acarretando consequências como violência, insegurança, desrespeito e ameaças ${ }^{(7)}$.

A vida do alcoolista é marcada por uma sucessão de episódios de crise, devido à convivência com os efeitos físicos e psíquicos fornecidos pelo consumo da bebida alcoólica. É comum a exposição social dessas pessoas e o envolvimento em acidentes de trânsito, isolamento, perdas econômicas e sociais e principalmente familiares, quando relacionadas ao cônjuge e aos filhos $^{(8)}$.

Os filhos de um alcoolista estão envolvidos também nos inúmeros conflitos familiares causados pela dependência. Crianças e adolescentes podem responder com comportamentos não saudáveis para o convívio familiar; assim, é comum perceber irritabilidade, isolamento, agressividade, frustração, angústia e dificuldade de comunicação $^{(8)}$.

Nesse contexto de crise, percebe-se a propensão ao envolvimento dos filhos com o álcool, com consequente abandono das atividades cotidianas e queda no rendimento escolar, acarretando, no âmbito familiar, situações cotidianas de desgaste, sobrecarga, desentendimento e agressividade, angústia, podendo requerer necessidades especiais de suporte, tornando a degradação familiar um dos maiores prejuízos adquiridos a partir da instalação da dependência.

Um estudo realizado com crianças corroborou a contribuição da dependência química para o desenvolvimento de sofrimento psíquico nesse grupo populacional, em que foram relatados sinais de autoestima baixa, timidez, insegurança, sintomas depressivos e isolamento, o que afeta, negativamente, a manutenção dos vínculos e das relações interpessoais ${ }^{(9)}$.

Assim, para nortear este estudo, foram traçadas as seguintes questões: Qual a estimativa de adolescentes que habitam lares com familiares alcoolistas no município de João Pessoa, Paraíba? Quais os perfis sociodemográfico e familiar dos adolescentes que convivem com familiares alcoolistas?

Esses questionamentos surgiram a partir dos resultados apontados por pesquisas realizadas em todo território nacional nos últimos anos, em que se tem observado um crescimento preocupante de pessoas usuárias de álcool ${ }^{(10-11)}$.

Desse modo, diante da complexidade da problemática e da necessidade de se ampliar a construção de bancos de dados que possibilitem uma visão panorâmica dos efeitos da drogadição no contexto brasileiro, esta investigação tem como objetivo estimar o quantitativo de adolescentes que habitam lares com familiares alcoolistas em uma amostra representativa de adolescentes na cidade de João Pessoa, Paraíba, e caracterizar os perfis sociodemográfico e familiar dos adolescentes que convivem com familiares alcoolistas.

\section{Metodologia}

O presente estudo é do tipo transversal de base populacional, realizado no ano de 2011, nas 
séries de ensino médio, em 45 escolas públicas estaduais do município de João Pessoa, registradas na Secretaria de Educação e Cultura da Paraíba. Foram incluídas, na pesquisa, todas as instituições, com o objetivo de se obter uma representatividade satisfatória da amostra.

Foi delimitada a população de 21.214 estudantes matriculados nas séries de ensino médio da rede estadual, que estivessem na faixa etária de 14 a 19 anos, tanto do sexo masculino quanto feminino, e residentes no município de João Pessoa.

Para a seleção dos adolescentes investigados, procedeu-se um cálculo amostral feito com o uso do sample size do programa OPEN EPI (Open Source Statistics for Public Health) versão 2.3.1, adotando-se $\mathrm{P}=95 \%$, uma frequência da população estimada em $20 \%$, com margem de erro de $3 \%$.

Foi utilizada a técnica de amostragem sistemática, empregada por se disponibilizar de uma população distribuída de forma organizada.

Para selecionar os participantes da pesquisa, foi organizada uma tabela de quatro colunas, em ordem numérica, que distribuía o número de alunos matriculados por turma em cada escola em um banco de dados no Excel 2007. Na sequência, foi feita uma equação que determinava o tamanho do intervalo dado entre um entrevistado e outro (29), de acordo com a tabela listada em ordem numérica (do número 1 a 21.214), seguindo um banco de dados fornecido pela Secretaria de Educação e Cultura da Paraíba, que constava nome da escola, turma, turno e número de alunos matriculados, com base em senso escolar realizado anualmente.

Os critérios adotados neste estudo possibilitaram a obtenção de uma amostra autoponderada pelo tamanho das escolas e das turmas nos três turnos: manhã, tarde e noite.

Foram incluídos no estudo 715 adolescentes, que foram submetidos a dois instrumentos: o primeiro trata-se do CAGE-familiar, uma adaptação do CAGE, disposto em 5 questões que tem por finalidade identificar familiares alcoolistas entre os adolescentes investigados, adotando-se duas respostas afirmativas como ponto de corte. O segundo foi a aplicação do questionário sociodemográfico, para traçar o perfil dos adolescentes e das famílias daqueles que convivem com familiares alcoolistas.
Após a finalização da coleta de dados, os resultados foram organizados e dispostos em tabelas e na sequência, analisados pela estatística simples e descritiva.

Este estudo obedeceu todos os requisitos éticos propostos pela Resolução no 196/96 do Conselho Nacional de Saúde (CNS), que dispõe de normas e diretrizes regulamentadoras de pesquisa envolvendo seres humanos. Dessa forma, para os adolescentes menores de 18 anos, foi exigida a autorização dos pais por meio da assinatura do Termo de Consentimento Livre e Esclarecido - TCLE, permitindo a participação do menor no estudo. O projeto foi aprovado sob o parecer de protocolo de $n^{\circ} 066 / 11$.

\section{Resultados}

Com o emprego do CAGE-familiar, identificaram-se, entre os participantes do estudo, os adolescentes que convivem com familiares alcoolistas. Assim, de acordo com a Tabela 1, dos $715(100 \%)$ colaboradores, 242 (33,9\%) adolescentes apresentaram CAGE- familiar positivo, e $473(66,1 \%)$ deles obtiveram CAGE-familiar negativo.

Tabela 1 - Frequência de adolescentes de acordo com as respostas do questionário CAGE-familiar, segundo a situação de convívio com familiares alcoolistas. João Pessoa, PB, Brasil, 2011

\begin{tabular}{lcc}
\hline \multirow{2}{*}{ Variáveis } & \multicolumn{2}{c}{ Adolescentes } \\
\cline { 2 - 3 } & $\mathbf{n}$ & $\%$ \\
\hline Não convivem com familiares alcoolistas & 473 & 66,1 \\
Convivem com familiares alcoolistas & 242 & 33,9 \\
Total & 715 & 100,0 \\
\hline
\end{tabular}

Após a aplicação do questionário CAGEfamiliar, de acordo com a Tabela 2, observa-se a frequência dos familiares alcoolistas, segundo a ordem das perguntas. Entre os familiares citados, o pai prevaleceu como maioria nas quatro perguntas, observando-se que, na primeira pergunta, foi citado em 155 (52,8\%) respostas; na segunda, em 101 (50,2\%); na terceira, em 83 (51,2\%); e, na quarta, em 38 (44,2\%).

Na sequência, são apresentados os dados que mostram o perfil dos adolescentes que convivem com familiares alcoolistas a partir das variáveis sociodemográficas e familiares. 
De acordo com a Tabela 3, os adolescentes entrevistados, em sua maioria, são do sexo feminino $(62 \%)$. Com relação à variável idade, foram distribuídos de acordo com a faixa etária de 14 a 19 anos - um dos critérios adotados para a inclusão dos sujeitos no estudo. Nessa pesquisa, prevaleceu a idade de 17 anos (24,0\%). Em referência à variável raça/cor, a maior parte afirmou ser parda $(61,6 \%)$. Predominou o catolicismo $(43,0 \%)$ como a prática religiosa dos adolescentes; e estes, em sua grande maioria, não trabalham $(71,1 \%)$.
A Tabela 4 foi gerada para descrever as categorias referentes ao perfil familiar. Identificou-se então que, em relação à variável escolaridade dos pais, predominou o ensino fundamental para o pai $(43,0 \%)$ e para a mãe $(50,8 \%)$; não se evidenciaram diferenças relevantes relacionadas ao grau de instrução entre as diferentes classificações de gênero. A maioria das famílias é formada por 4 pessoas $(38,4 \%)$, prevalecendo o modelo familiar nuclear $(46,3 \%)$, em sua maioria, lideradas pelo pai $(43,8 \%)$, e dispondo de uma renda familiar variável de até 2 salários mínimos $(60,3 \%)$.

Tabela 2 - Distribuição dos familiares que fazem uso do álcool por sequência de perguntas do questionário CAGE-familiar. João Pessoa, PB, Brasil, 2011

\begin{tabular}{|c|c|c|c|c|}
\hline \multirow{2}{*}{ Perguntas cage familiar } & \multicolumn{4}{|c|}{ Familiares que fazem uso do álcool } \\
\hline & Pai & Mãe & Padrasto & Outros \\
\hline $\begin{array}{l}\text { 1-Você já sentiu que alguém em sua família deveria parar de beber? } \\
\text { Quem? }\end{array}$ & $\begin{array}{c}155 \\
(55,8 \%)\end{array}$ & $\begin{array}{c}17 \\
(6,1 \%)\end{array}$ & $\begin{array}{c}21 \\
(7,0 \%)\end{array}$ & $\begin{array}{c}85 \\
(30,6 \%)\end{array}$ \\
\hline $\begin{array}{l}\text { 2- Tem alguém em sua família que já se sentiu incomodado por } \\
\text { reclamações e críticas sobre o seu hábito de beber? Quem? }\end{array}$ & $\begin{array}{c}101 \\
(50,2 \%)\end{array}$ & $\begin{array}{c}15 \\
(7,5 \%)\end{array}$ & $\begin{array}{c}15 \\
(7,5 \%)\end{array}$ & $\begin{array}{c}70 \\
(34,8 \%)\end{array}$ \\
\hline $\begin{array}{l}\text { 3-Tem alguém em sua família que já se sentiu mal ou culpado por beber? } \\
\text { Quem? }\end{array}$ & $\begin{array}{c}83 \\
(51,2 \%)\end{array}$ & $\begin{array}{c}16 \\
(9,9 \%)\end{array}$ & $\begin{array}{c}12 \\
(7,4 \%)\end{array}$ & $\begin{array}{c}51 \\
(31,5 \%)\end{array}$ \\
\hline 4-Tem alguém em sua família que já tomou bebida em jejum? Quem? & $\begin{array}{c}38 \\
(44,2 \%)\end{array}$ & $\begin{array}{c}9 \\
(10,5 \%)\end{array}$ & $\begin{array}{c}3 \\
(3,5 \%)\end{array}$ & $\begin{array}{c}36 \\
(41,9 \%)\end{array}$ \\
\hline
\end{tabular}

Tabela 3 - Distribuição dos adolescentes que convivem com familiares alcoolistas segundo as variáveis sociodemográficas. João Pessoa, PB, Brasil, 2011

\begin{tabular}{|c|c|c|}
\hline \multirow{2}{*}{$\begin{array}{l}\text { Características } \\
\text { sociodemográficas }\end{array}$} & $\mathbf{n}$ & $\%$ \\
\hline & $\mathrm{n}=\mathbf{2 4 2}$ & 100 \\
\hline \multicolumn{3}{|l|}{ Sexo } \\
\hline Masculino & 92 & 38,0 \\
\hline Feminino & 150 & 62,0 \\
\hline \multicolumn{3}{|l|}{ Idade } \\
\hline 14 & 12 & 5,0 \\
\hline 15 & 39 & 16,1 \\
\hline 16 & 46 & 19,0 \\
\hline 17 & 58 & 24,0 \\
\hline 18 & 49 & 20,2 \\
\hline 19 & 38 & 15,7 \\
\hline \multicolumn{3}{|l|}{ Raça/cor } \\
\hline Branca & 59 & 24,4 \\
\hline Parda & 149 & 61,6 \\
\hline Preta & 34 & 14,0 \\
\hline \multicolumn{3}{|l|}{ Religião } \\
\hline Sem religião & 47 & 19,4 \\
\hline Católico & 104 & 43,0 \\
\hline Evangélico & 77 & 31,8 \\
\hline Outros & 14 & 5,8 \\
\hline \multicolumn{3}{|l|}{ Atividade remunerada } \\
\hline Não trabalha & 172 & 71,1 \\
\hline Trabalha & 70 & 28,9 \\
\hline
\end{tabular}

Tabela 4 - Distribuição dos adolescentes que convivem com familiares alcoolistas, segundo as características familiares. João Pessoa, PB, Brasil, 2011

\begin{tabular}{|c|c|c|}
\hline \multirow{2}{*}{ Perfil familiar } & $\mathbf{n}$ & $\%$ \\
\hline & 242 & 100 \\
\hline \multicolumn{3}{|l|}{ Escolaridade do pai } \\
\hline Ensino superior & 14 & 5,8 \\
\hline Ensino médio & 87 & 36,0 \\
\hline Ensino fundamental & 104 & 43,0 \\
\hline Não alfabetizado & 12 & 5,0 \\
\hline Sem informação & 25 & 10,3 \\
\hline \multicolumn{3}{|l|}{ Escolaridade da Mãe } \\
\hline Ensino superior & 17 & 7,0 \\
\hline Ensino médio & 81 & 33,5 \\
\hline Ensino fundamental & 123 & 50,8 \\
\hline Não alfabetizado & 7 & 2,9 \\
\hline Sem informação & 14 & 5,8 \\
\hline \multicolumn{3}{|c|}{ Número de moradores da casa } \\
\hline Até 2 pessoas & 17 & 7,0 \\
\hline 3 pessoas & 42 & 17,4 \\
\hline 4 pessoas & 93 & 38,4 \\
\hline 5 pessoas & 50 & 20,7 \\
\hline Mais que 6 pessoas & 40 & 16,5 \\
\hline \multicolumn{3}{|l|}{ Tipo de família } \\
\hline Nuclear & 112 & 46,3 \\
\hline Extensa & 73 & 30,2 \\
\hline Monoparental & 34 & 14,0 \\
\hline Recomposta & 23 & 9,5 \\
\hline
\end{tabular}


Tabela 4 - continuação

\begin{tabular}{lcc}
\hline \multirow{2}{*}{ Perfil familiar } & $\mathbf{n}$ & \% \\
\cline { 2 - 3 } & $\mathbf{2 4 2}$ & $\mathbf{1 0 0}$ \\
\hline Chefe da família & & \\
Pai & 106 & 43,8 \\
Mãe & 77 & 31,8 \\
Pai/mãe & 13 & 5,4 \\
Outros & 43 & 17,8 \\
Sem informação & 3 & 1,2 \\
Renda familiar & & \\
Até 2 salários mínimos & 146 & 60,3 \\
3 a 4 salários mínimos & 58 & 24,0 \\
$>5$ salários mínimos & 24 & 9,9 \\
Sem informação & 14 & 5,8 \\
\hline
\end{tabular}

Fonte: pesquisa direta

\section{Discussão}

O consumo do álcool no Brasil tem se mostrado preocupante, são crescentes os números de usuários e de problemas em decorrência ao uso abusivo dessa substância. No I Levantamento Nacional sobre os padrões de consumo de álcool na população brasileira, verificou-se que 52\% dos brasileiros podem ser classificados como bebedores; entre eles, $27 \%$ fazem uso ocasional ou raro do álcool e $25 \%$ representam aquelas pessoas que fazem uso da substância pelo menos uma vez por semana ${ }^{(10)}$.

De acordo com esses resultados, a grande maioria das famílias brasileiras convive com pessoas que fazem uso frequente do álcool. Tais pessoas são, frequentemente, pais de família, desempregados ou subempregados e jovens adolescentes sem inserção na escola ou no mercado de trabalho. A problemática também envolve adultos - homens e mulheres - independentemente da classe social, uma vez que cada vez mais se banaliza o consumo exagerado do álcool e suas consequências, resultando em famílias fragilizadas.

$\mathrm{O}$ ato de beber tem profundo impacto sobre a família, seja no aspecto considerado uso nocivo ou dependência. Embora o alcoolismo seja um mal que atinge as pessoas de forma individual, ao mesmo tempo, ele destrói seus vínculos sociais e os afetivos, prejudica seu local de trabalho e sua família, tornando-a, dessa forma, vítima do álcool pelos efeitos que lhes é causado, dentre eles o sofrimento psíquico dos seus familiares.
Nesta pesquisa, identificou-se que, dentre os familiares usuários de álcool, o pai foi o mais mencionado, sendo citado em todas as respostas do questionário CAGE-familiar. Em outro estudo realizado com filhos de dependentes químicos em São Paulo, o pai foi referenciado na maioria das vezes, como o dependente químico e tendo o álcool como a substância de escolha ${ }^{(12)}$. No México, uma pesquisa realizada sobre depressão em adolescentes, verificou-se que o abuso do álcool foi predominante pelo pai com $23 \%$, contra $3 \%$ pela mãe ${ }^{(13)}$.

Essas referências comprovam evidências de outros estudos em que o uso do álcool é prevalente em pessoas do sexo masculino. Como foi verificado em um inquérito nacional, $65 \%$ dos homens entrevistados, maiores de 18 anos, bebem $^{(10)}$. E em investigação realizada no Estado de São Paulo, a prevalência do consumo de álcool entre os adultos do sexo masculino foi de $52,9 \%{ }^{(14)}$.

Baseado na hipótese de que conviver com um familiar alcoolista é sempre um desafio, visto que existe a possibilidade de um ou mais de seus membros manifestarem sinais de adoecimento psíquico, decorrente da relação estabelecida entre o indivíduo e o álcool, compreende-se que a dependência química passa a regular as relações familiares; assim, os referidos membros passam a demonstrar sinais de codependência, uma patologia que se manifesta pela relação de dependência estabelecida entre as pessoas.

Com a compreensão de que a adolescência representa uma grande parcela da população mundial, e é nessa fase da vida que as pessoas tornam-se vulneráveis, expostas a situações de risco, dar-se a importância de se conhecer os perfis sociodemográfico e familiar dos jovens que convivem com familiares alcoolistas.

A predominância de mulheres neste estudo é um reflexo dos parâmetros apresentados pelo último censo, que informa maior parte da população brasileira ser composta por pessoas do sexo feminino e que corresponde a $51 \%$ do total. Com relação à variável raça/cor, de acordo com os últimos resultados, no geral, a população brasileira autorreferiu como da cor branca, porém, no que se refere à população de adolescentes do país, esses, em sua maioria, são da cor parda, com $48,9 \%$, comprovando a semelhança de dados encontrados nesta investigação ${ }^{(15)}$. 
Quanto à determinante religião, no Brasil, esta tem sido uma variável apontada com bastante flexibilidade nos últimos tempos. Embora o número crescente de evangélicos no país tenha sido um dos dados mais referenciados nos últimos anos, evidencia-se a predominância do catolicismo, como foi verificado entre os participantes deste estudo ${ }^{(15)}$.

O espaço escolar foi definido como local de pesquisa neste estudo. Esta é uma das justificativas que comprovam a maioria no número de indivíduos que não trabalham. Segundo dados dos indicadores sociais da população brasileira, a maioria dos adolescentes que estão na escola não está inserida no mercado de trabalho, mesmo considerando o crescimento, segundo os dados do censo 2010, que mostram que $3,5 \%$ da população de 10 a 20 anos do país, atualmente possuem algum tipo de rendimento ${ }^{(15)}$.

Esse resultado assemelha-se ao apresentado no levantamento sobre padrão de consumo de álcool na população brasileira, que revelou um comparativo entre os sujeitos participantes do estudo em duas faixas etárias, de 14 a 17 anos e de 18 anos ou mais, no qual os autores identificaram que quase um terço dos adolescentes pertencia à população economicamente ativa. Embora o número de adolescentes que trabalham seja inferior ao dos adultos, este resultado mostra-se preocupante, pois indica que muitos já estão inseridos no mercado de trabalho, e este é um agravante que limita a permanência desse grupo na escola ${ }^{(10)}$.

Em referência à escolaridade do pai e da mãe dos entrevistados, os resultados corroboram com os últimos dados publicados sobre os indicadores sociais brasileiros que apresentam a média de 7 anos de estudo no país, representando que o grau de escolaridade para a maioria da população brasileira limita-se a não conclusão do ensino fundamental ${ }^{(15)}$.

Esses dados denotam o quadro educacional atual dos países em desenvolvimento, que apresentam, entre suas características sociodemográficas, alto índice de pobreza e baixo grau de escolaridade, que ainda é apontada como uma das variáveis sociodemográficas que contribuem para a exposição a situações de risco. O processo de globalização e as exigências do mercado de trabalho, com a qualificação profissional das pessoas, têm gerado discursos a respeito do processo educacional no Brasil que vem passando por avaliações constantes, tendo como um de seus objetivos garantir a permanência dos jovens na escola ${ }^{(16)}$.

O perfil geral das famílias dos adolescentes envolvidos neste estudo é semelhante aos divulgados pelo Instituto Brasileiro de Geografia e Estatística ${ }^{(15)}$, uma vez que este descreve um número reduzido de pessoas por família (três pessoas por residência), predominando o modelo familiar nuclear, sendo esse chefiado por homens com uma renda de até dois salários mínimos.

A drogadição tem se apresentado como um dos problemas sociais mais debatidos nos últimos tempos, dentre eles, o alcoolismo. Essa problemática trata-se de uma ameaça global, cujo enfrentamento não pode ser pontual sob a forma exclusiva de ações assistenciais, mas sim por estratégias eficientes com enfrentamento amplo e eficaz ${ }^{(17)}$.

\section{Considerações finais}

A partir das leituras realizadas para a confrontação dos dados deste estudo, tornou-se evidente a participação negativa que a bebida alcoólica tem no cotidiano das famílias, levando em consideração que, depois da instalação da situação de dependência, as relações familiares começam a ser reguladas pelo estado de saúde e sobriedade do dependente químico.

Todos esses eventos geram um ambiente familiar tenso, desgastado, com necessidade de fortalecimento dos laços de afetividade. Para tanto, não há comportamento universal, cada família reage de forma diferente, principalmente devido às particularidades relacionadas à situação financeira, escolaridade, religiosidade e rede de apoio.

Em se tratando de adolescentes e sua exposição diária ao consumo de substâncias psicoativas, um familiar dependente pode influenciar no desenvolvimento do consumo abusivo, bem como no acometimento por sequelas emocionais, que acompanharão o adolescente por toda vida, acarretando danos em todas as esferas, tanto familiar quanto social.

Com base nos resultados, compreende-se a necessidade de um crescente planejamento e implementação de serviços de saúde especializados no atendimento aos adolescentes e familiares afetados pela dependência química, especialmente de drogas lícitas, como o álcool. 
Nesse contexto, são indispensáveis as intervenções eficazes dos profissionais de saúde, enfatizando a contribuição da enfermagem caracterizada por ser a ciência do cuidado humano, atuando em estratégias de promoção da saúde, prevenção, reabilitação e tratamento de agravos, exercendo papel fundamental também na atenção psicossocial a familiares cuidadores e adolescentes expostos ao convívio com dependentes químicos.

\section{Referências}

1. Alves VS. Modelos de atenção à saúde de usuários de álcool e outras drogas: discursos políticos, saberes e práticas. Cad Saúde Pública. 2009;25(11):2309-19.

2. Anthony JC. Consumo nocivo de álcool: dados epidemiológicos mundiais. In: Andrade AG, Anthony JC, Silveira CM, Organizadores. Álcool e suas consequências: uma abordagem multiconceitual. Barueri: Minha Editora; 2009. p. 1-36.

3. Ministério da Saúde (BR). Secretaria de Atenção à Saúde. Saúde mental e atenção básica: o vínculo e o diálogo necessários. Brasília: MS; 2007.

4. Rodrigues DS, Backes DS, Freitas HMB, Zamberlan C, Gelhen MH, Colomé JS. Conhecimentos produzidos acerca do crack: uma incursão nas dissertações e teses brasileiras. Ciênc Saúde Coletiva. 2012;17(5):1247-58.

5. Organização Mundial da Saúde. Transtornos devido ao uso de substâncias. In: Organização Pan-Americana da Saúde \& Organização Mundial da Saúde, Organizadores. Relatório sobre a saúde no mundo. Saúde Mental: nova concepção, nova esperança. Brasília: Gráfica Brasil; 2001. p. 58-61.

6. Sousa PF, Ribeiro LCM, Melo JRF, Maciel $\mathrm{SC}$, Oliveira MX. Dependentes químicos em tratamento: estudo sobre a motivação para a mudança. Temas Psicol. 2013;21(1):259-68.

7. Ferreira MO Filha, Sá ANP, Rocha IA, Silva VCL, Souto CMRM, Dias MD. Alcoolismo no contexto familiar: estratégias de enfrentamento de idosas usuárias da terapia comunitária. Rev Rene. 2012;13(1):26-35.

8. Jorge MSB, Lopes CHAF, Sampaio CF, Souza LV, Silva MSJ, Alves MS. Alcoolismo nos contextos social e familiar: análise documental à Luz de Pimentel. Rev Rene. 2007;8(3):26-33.

9. Reinaldo MAS, Pillon SC. Alcohol effects on family relations: a case study. Rev. Latino-Am. Enfermagem. 2008;16(n.esp):1-6.

10. Laranjeira R, Pinsky I, Zaleski M, Caetano $\mathrm{R}$, organizadores. I Levantamento Nacional sobre os padrões de consumo de álcool na população brasileira. Brasília: Secretaria Nacional Antidrogas; 2007.

11. Carlini EA. II Levantamento domiciliar sobre o uso de drogas psicotrópicas no Brasil: estudo envolvendo as 108 maiores cidades do país, 2005. São Paulo: Secretaria Nacional Antidrogas (SENAD)/Gabinete de Segurança InstitucionalPresidência da República/ CEBRID/ UNIFESP; 2006. 468 p.

12. Fligie N, Fontes A, Moraes E, Paya R. Filhos de dependentes químicos com fatores de risco bio-psicossociais: necessitam de um olhar especial? Rev Psiq Clín. 2004;31(2):53-62.

13. Barrientos-Acosta V, Mendoza-Sánchez HF, Sainz-Vázquez L, Pérez-Hernández C, GilAlfaro I, Soler-Huerta E. Depresión y tipología familiar en un grupo de adolescentes mexicanos. Arch Medicina Familiar. 2010;12(3):69-76.

14. Guimarães VV, Florindo AA, Stopa SR, César CLG, Barros MBA, Carandina L, et al. Consumo abusivo e dependência de álcool em população adulta no Estado de São Paulo, Brasil. Rev Bras Epidemiol. 2010;13(2):314-25.

15. Instituto Brasileiro de Geografia e Estatística (IBGE). Síntese de Indicadores Sociais: uma análise das condições de vida da população brasileira 2010. Rio de Janeiro: IBGE; 2010. n. 27. 16. Silva MR, Pelissari LB, Steimbach AA. Juventude, escola e trabalho: permanência e abandono na educação profissional técnica de nível médio. Educ Pesqui. 2013;39(2):403-17.

17. Gurgel WB, Mochel AG, Carvalho Filha FSS. O abuso do álcool como problema político: análise das estratégias políticas de assistência ao consumidor abusivo de álcool no Brasil contemporâneo. Cad Pesq. 2010;17(1):53-63. 